\section{Comportamiento clínico y factores asociados a mortalidad temprana en una cohorte de pacientes chilenos con vasculitis asociadas a anticuerpos anti citoplasma de neutrófilos (VAA)}

\author{
DANIELA VARGAS ${ }^{1}$, ANNELISE GOECKE $^{1}$, HÉCTOR GATICA ${ }^{1}$, \\ ARIEL CASTRO LARA ${ }^{2, a}$, PAMELA WURMANN ${ }^{1}$
}

\section{Variables associated with mortality in 103 patients with anti-neutrophil cytoplasmic antibodies associated vasculitis}

Background: Cumulative survival in patients with anti-neutrophil cytoplasmic antibodies (ANCA) associated vasculitis (VAA) is 88 and 78\% at 1 and 5 years, respectively. Despite this, mortality continues to be 2.7 times higher than the general population. Differences in the clinical profile of VAA in different ethnicities have been observed. Aim: To identify factors at the time of diagnosis, associated with mortality at one year of follow-up and to describe the clinical characteristics of these patients. Material and Methods: We identified in local databases and reviewed clinical records of patients with VAA with at least one year of follow up in a clinical hospital. Demographic and laboratory parameters and clinical activity scores were analyzed. Results: Of 103 patients with VAA identified, 65 met the inclusion criteria and were analyzed. Their age ranged from 45 to 63 years and 56\% were women. Thirty-five patients (54\%) were diagnosed as granulomatosis with Polyangiitis (GPA) and 30 patients (46\%) with Microscopic Polyangiitis (MPA). The frequency of renal disease was 53\% and pulmonary involvement occurred in 72\%. At one year of follow-up 11 patients died resulting in a mortality of $17 \%$. Seven patients died within three months after diagnosis. MPO ANCA were more common than PR3 ANCA. In the multivariate analysis, the presence of ophthalmological involvement, lung kidney syndrome and a Five Factor Score (FFS) of 1 or more were independent factors associated with mortality at one year. Conclusions: In these patients, pulmonary manifestations predominate. Lung kidney syndrome, ophthalmological involvement and a FFS score $\geq 1$ were associated with mortality.

(Rev Med Chile 2020; 148: 755-761)

Key words: Antibodies; Antibodies, Antineutrophil Cytoplasmic; Mortality; Vasculitis.

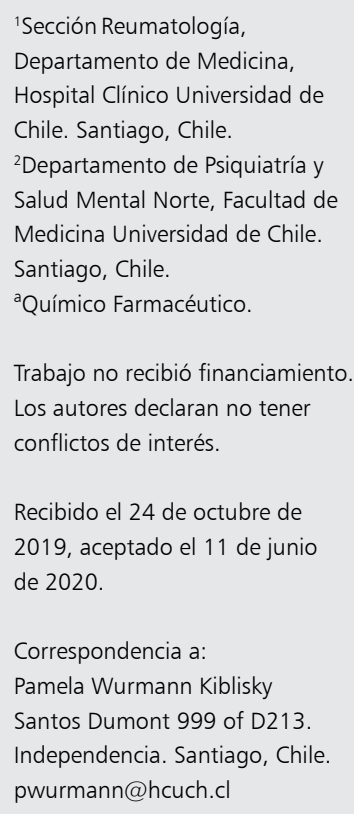

I as vasculitis asociadas a ANCA (VAA), son un grupo de enfermedades que se caracterizan por inflamación necrotizante de la pared de pequeños vasos y la presencia de anti- cuerpos anti citoplasma de neutrófilos (ANCA) circulantes.

Sus manifestaciones clínicas son variables, pudiendo presentarse como compromiso aislado 
de un órgano o una enfermedad multisistémica con riesgo vital.

Antes de la introducción de las terapias vigentes se describía una mortalidad de $80 \%$ al año. Actualmente la sobrevida acumulada en pacientes con VAA, se ha estimado en $88 \%$ al año y $78 \%$ a 5 años ${ }^{1}$. Pese a esto, la mortalidad continúa siendo 2,7 veces mayor que la población general ${ }^{2}$.

Distintas herramientas han sido desarrolladas para evaluar la actividad y el riesgo de muerte de las VAA como el Birmingham Vasculitis Activity Score (BVAS) y el Five Factor Score (FFS) ${ }^{3,4}$. Se han establecido como factores predictores de muerte al diagnóstico: la edad avanzada, la velocidad de filtración glomerular (VFG) $<15 \mathrm{ml} / \mathrm{min}$, el BVAS elevado, la hemoglobina baja y el recuento elevado de leucocitos ${ }^{1}$.

Se han reportado diferencias en el perfil clínico de las VAA asociado a distintas etnias ${ }^{5,6}$. Existen escasos datos respecto a las características y al pronóstico en población latinoamericana. Sreih et al. reportaron que los pacientes hispanoamericanos tienen una presentación más severa y peores índices de daño que la población caucásica ${ }^{7}$. El único dato reportado de una cohorte chilena observó una mortalidad global del $17 \%$ para GPA y $18 \%$ PAM. Los valores de creatinina mayor a $2 \mathrm{mg} / \mathrm{dL}$ fueron predictores de mortalidad ${ }^{8}$.

Si bien, las VAA son infrecuentes, su curso potencialmente grave y su alta mortalidad sin un tratamiento adecuado, hacen necesario incrementar la información respecto a su evolución clínica y los factores asociados a mortalidad.

Este trabajo tiene como objetivos describir el comportamiento clínico en pacientes chilenos con vasculitis ANCA e identificar los factores al diagnóstico que se asocian a muerte al año.

\section{Materiales y Métodos}

Mediante las bases locales para búsqueda, se identificaron los pacientes con diagnóstico de vasculitis ANCA en el Hospital Clínico de la Universidad de Chile entre los años 2000 y 2016.

Se incluyeron pacientes con diagnóstico de GPA o PAM que tuvieran al menos un año de seguimiento en el caso de los no fallecidos. Se excluyeron pacientes con otras vasculitis, con diagnóstico realizado en otro centro, aquellos sin datos clínicos y/o de laboratorio disponibles al diagnóstico o que no cumplieran con el plazo de seguimiento establecido.

En forma retrospectiva se analizaron las fichas clínicas. Se obtuvo datos sociodemográficos y el registro de síntomas constitucionales, compromiso cutáneo, oftalmológico, otorrinolaringológico, pulmonar, renal, neurológico y músculo esquelético. Se definió glomerulonefritis (GN) como la presencia de al menos 2 sedimentos de orina inflamatorios (eritrocitos, leucocitos y proteínas mayor al rango normal), en ausencia de infección urinaria. Se definió insuficiencia renal como velocidad de filtración glomerular $<60 \mathrm{ml} / \mathrm{min}$. El término síndrome riñón pulmón se utilizó cuando el paciente presentaba GN asociado a hemorragia alveolar.

Se consideraron los valores de laboratorio del debut de la enfermedad como hemograma, velocidad de eritrosedimentación (VHS), proteína $C$ reactiva (PCR), creatinina plasmática y determinación de ANCA por IFI y ELISA. También se analizó el tratamiento de inducción recibido por cada paciente.

En todos los pacientes se calculó al diagnós-

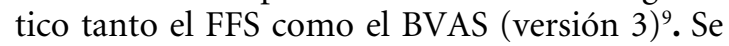
registró la mortalidad al año y la causa de muerte en base a los registros clínicos y la página web del registro civil.

Los resultados se presentan en medidas de posición central (medianas) y medidas de dispersión (percentiles 25-75) para variables cuantitativas $\mathrm{y}$ en números absolutos $\mathrm{y}$ frecuencias relativas para las cualitativas. Las curvas de sobrevida se compararon mediante las pruebas de log-rank test y Wilcoxon-Gehan-Breslow, considerándose significativo un $p$ value $<0,05$.

Para estimar factores asociados a mortalidad al año, se realizó un análisis de regresión de Cox bivariado y multivariado. Se comprobó la condición de riesgos proporcionales mediante el método de los residuos de Schoenfeld y el ajuste del modelo mediante el criterio de Akaike.

El modelo multivariado se ajustó con el método hacia atrás (stepwise), incorporando en el modelo final todas aquellas variables que resultaran tener un $p$ value $<0,2$.

Se utilizó el programa estadístico Stata v12.1

Este estudio fue aprobado por el comité de ética del Hospital Clínico de la Universidad de Chile. 


\section{Resultados}

Se encontraron 103 pacientes con diagnóstico de VAA. 65 cumplieron con los criterios de inclusión. La mayoría fueron mujeres y la mediana de edad fue de 53 años. GPA y PAM tuvieron una frecuencia similar, $35(53,85 \%)$ y 30 pacientes $(46,2 \%)$ respectivamente.

En relación a la presentación clínica los síntomas constitucionales fueron los más frecuentes (fiebre, baja de peso), seguidos del compromiso pulmonar, músculo esquelético (artralgias, artritis, mialgias), otorrinolaringológico (otitis, sinusitis, estenosis subglótica) y renal. Dentro del compromiso pulmonar, los nódulos, la hemorragia alveolar y la enfermedad pulmonar difusa (EPD) tuvieron una frecuencia similar. Como manifestación renal, la glomerulonefritis (GN) con IR (insuficiencia renal) fue más frecuente que la GN sin IR. Más de un cuarto de los pacientes presentaron síndrome riñón pulmón. El compromiso oftalmológico estuvo dado principalmente por epiescleritis y esclerouveitis, objetivado en 16 pacientes; 3 presentaron tumor inflamatorio de la órbita y 4 dacrioadenitis. La manifestación cutánea más frecuente fue púrpura palpable, observada en nueve pacientes. En cuanto al compromiso neurológico, sólo se observó afección del sistema nervioso periférico, siendo el más frecuente la polineuropatía sensitivo motora objetivada en siete pacientes. De los cuatro pacientes con compromiso cardiológico al diagnóstico, tres presentaron pericarditis y uno miocarditis. En un paciente se objetivó hemorragia digestiva baja como manifestación de vasculitis. La mediana de BVAS fue 20 (15-24) y de FFS 1 (0-1) (Tabla 1).

Respecto a los hallazgos de laboratorio (Tabla 2), las alteraciones más frecuentes fueron anemia $(70,8 \%)$ y elevación de reactantes de fase aguda, principalmente PCR (92\%). En 64 pacientes se realizó determinación de ANCA por IFI, la mayoría tuvo patrón P ANCA (33 pacientes) y 4 resultaron negativos. En 57 pacientes se hizo determinación por técnica de ELISA, siendo más frecuentes los con MPO positivo. Aquellos seronegativos tuvieron confirmación histológica de vasculitis. En 24 pacientes se realizó biopsia: 6 de pulmón, 6 de riñón, 4 de vía aérea superior, 2 de nervio sural, 2 de piel y 4 en otros tejidos (testículo, órbita, tumor de oído y hematoma subdural), evidenciando vasculitis de pequeño vaso en 13.
Tabla 1. Características demográficas y clínicas al diagnóstico

\begin{tabular}{|c|c|}
\hline & n $\quad(\%)$ \\
\hline Total de pacientes & $65(100)$ \\
\hline $\begin{array}{l}\text { Edad al momento del diagnóstico, } \\
\text { mediana (p25 - p75) }\end{array}$ & $53(45-63)$ \\
\hline Sexo femenino & $38(58,5)$ \\
\hline Síntomas constitucionales & $51(78,5)$ \\
\hline Baja de peso & $33(50,8)$ \\
\hline Fiebre & $37(56,9)$ \\
\hline Pulmonar & $47(72,3)$ \\
\hline Hemorragia & $21(32,3)$ \\
\hline Nódulos & $21(32,3)$ \\
\hline Enfermedad pulmonar difusa & $17(26,2)$ \\
\hline Músculo esquelético & $40(61,5)$ \\
\hline Artralgias & $14(21,5)$ \\
\hline Artritis & $21(32,3)$ \\
\hline Mialgias & $17(26,2)$ \\
\hline Otorrinolaringológico & $38(58,5)$ \\
\hline Sinusitis & $30(46,2)$ \\
\hline Epistaxis & $15(23,1)$ \\
\hline Estenosis subglótica & $4(6,2)$ \\
\hline $\begin{array}{l}\text { Nariz en silla de montar/perforación } \\
\text { tabique nasal }\end{array}$ & $5(7,7)$ \\
\hline Otitis/mastoiditis & $11(16,9)$ \\
\hline Renal & $35(53,8)$ \\
\hline Glomerulonefritis sin insuficiencia renal & $5(7,7)$ \\
\hline Glomerulonefritis con insuficiencia renal & $26(40)$ \\
\hline Oftalmológico & $23(35,4)$ \\
\hline Epiescleritis/esclerouveitis & $16(24,6)$ \\
\hline Tumor inflamatorio de la orbita & $3(4,6)$ \\
\hline Dacrioadenitis & $4(6,2)$ \\
\hline Neurológico & $18(27,7)$ \\
\hline Polineuropatía sensitivo motora & $7(10,8)$ \\
\hline Mononeuritis múltiple & $5 \quad(7,7)$ \\
\hline Síndrome riñón-pulmón* & $17(26,2)$ \\
\hline Piel & $16(24,6)$ \\
\hline Púrpura palpable & $9(13,8)$ \\
\hline Lívedo reticularis & $4(6,2)$ \\
\hline Cardiaco & $4(6,2)$ \\
\hline Pericarditis & $3(4,6)$ \\
\hline Miocarditis & $1(1,5)$ \\
\hline
\end{tabular}

*Glomerulonefritis asociado a hemorragia alveolar. 
En cuanto al tratamiento de inducción, el $100 \%$ de los pacientes recibió corticoides (Tabla 3) y el inmunosupresor más utilizado fue ciclofosfamida, seguido de azatioprina, metrotrexato y rituximab. Tres pacientes murieron antes de re-

\section{Tabla 2. Marcadores inmunológicos y de laboratorio clínico al diagnóstico}

\begin{tabular}{|lc|}
\hline & Mediana (p25-p75) \\
\hline Hemoglobina $(\mathrm{g} / \mathrm{dl})$ & $11,3(9,8-12,3)$ \\
Recuento leucocitos/mL $\mathrm{mL}^{3}$ & $11500(8.940-16.000)$ \\
Recuento plaquetas/mL $\mathrm{mL}^{3}$ & $443.000(323.000-532.000)$ \\
\hline PCR $(\mathrm{mg} / \mathrm{l})$ & $145(60,5-215)$ \\
VHS $(\mathrm{mm} / \mathrm{h})$ & $78(61-113)$ \\
Creatinina plasmática $(\mathrm{mg} / \mathrm{dl})$ & $0,9(0,7-1,6)$ \\
\hline & $\mathbf{n}(\mathbf{( \% )}$ \\
ANCA por IFI positivo & $60(92,3)$ \\
ANCA P & $33(50,8)$ \\
ANCA C & $25(38,5)$ \\
ANCA atípico & $2(3,1)$ \\
ANCA por ELISA positivo & $51(78,5)$ \\
Anti-MPO & $31(47,7)$ \\
Anti-PR3 & $20(30,8)$ \\
\hline
\end{tabular}

ANCA: anticuerpos anti citoplasma de neutrófilos; IFI: inmunofluorescencia indirecta; ELISA: inmunoabsorción ligado a enzimas; MPO: mieloperoxidasa; PR3: proteinasa 3; PCR: proteína $\mathrm{C}$ reactiva VHS: velocidad de eritrosedimentación. cibir terapia de inducción y en dos no se encontró información respecto al tratamiento. En cuatro pacientes se realizó recambio plasmático.

$\mathrm{Al}$ año de seguimiento la mortalidad fue $16,9 \%$, con 11 pacientes fallecidos, 7 de ellos antes del tercer mes de diagnóstico. La causa de muerte fue infección en 5 pacientes, vasculitis activa en 4 , infarto agudo al miocardio en uno y estatus epiléptico no convulsivo en uno.

Las curvas de sobrevida según la edad, la presencia de compromiso pulmonar, renal, síndrome riñón pulmón, valor de FFS y BVAS se muestran en la Figura 1.

En el análisis multivariado (Tabla 4), la presencia del compromiso oftalmológico, del síndrome riñón pulmón y del valor de FFS $>0=a 1$ resultaron ser factores independientes asociados a la mortalidad al año $(\mathrm{p}<0,05)$.

\section{Tabla 3. Tratamiento de inducción}

\begin{tabular}{|lc|}
\hline \multicolumn{1}{|l|}{ n (\%) } \\
Corticoides & $35(53,8)$ \\
Bolos de metilprednisolona & $27(41,5)$ \\
Prednisona $1 \mathrm{mg} / \mathrm{kg}$ & $3(4,6)$ \\
Prednisona $<1 \mathrm{mg} / \mathrm{kg}$ & \\
Inmunosupresores & $53(81,5)$ \\
Ciclofosfamida oral o iv & $6(9,2)$ \\
Metrotrexato o azatioprina & $1(1,5)$ \\
Rituximab & \\
\hline
\end{tabular}

Tabla 4. Factores relacionados a muerte al año de acuerdo al análisis univariado y multivariado

\begin{tabular}{|c|c|c|c|c|c|c|}
\hline \multirow[b]{2}{*}{ Factor } & \multicolumn{4}{|c|}{ Análisis univariado } & \multicolumn{2}{|c|}{ Análisis multivariado } \\
\hline & $\begin{array}{l}\text { Vivos } \\
\text { n (\%) }\end{array}$ & $\begin{array}{c}\text { Fallecidos } \\
\text { n (\%) }\end{array}$ & $\begin{array}{c}\text { HR } \\
\text { (IC 95\%) }\end{array}$ & P value & $\begin{array}{c}\text { HR } \\
\text { (IC 95\%) }\end{array}$ & P value \\
\hline Edad $>0=65$ & $9(16,7)$ & $5(45,5)$ & $3,5(1,1-11,5)$ & 0,039 & $3,1(0,8-11,7)$ & 0,105 \\
\hline Compromiso pulmonar & $37(68,5)$ & $10(90,91)$ & $4,3(0,6-33,3)$ & 0,165 & & \\
\hline Compromiso renal & $24(46,3)$ & $10(90,9)$ & $9,7(1,2-76,1)$ & 0,030 & & \\
\hline Compromiso oftalmológico & $17(31,5)$ & $6(54,55)$ & $2,5(0,8-8,3)$ & 0,128 & $9,4(2,3-38,9)$ & 0,002 \\
\hline Síndrome riñón-pulmón & $10(18,5)$ & $7(63,6)$ & $6,2(1,8-21,2)$ & 0,004 & $4,94(1,3-18,9)$ & 0,020 \\
\hline Anemia & $36(66,7)$ & $10(90,9)$ & $4,4(0,6-34,5)$ & 0,157 & & \\
\hline Leucocitosis & $28(51,9)$ & $6(54,55)$ & $2,4(0,6-9,0)$ & 0,198 & & \\
\hline Creatinina $>0=1,5$ & $13(24,1)$ & $7(63,65)$ & $4,5(1,3-15,4)$ & 0,017 & & \\
\hline $\mathrm{FFS}>0=1$ & $25(46,3)$ & $10(90,91)$ & $9,7(1,2-76,1)$ & 0,030 & $9,36(1,0-86,5)$ & 0,049 \\
\hline
\end{tabular}

FFS: five factor score; IC: intervalo de confianza; HR: hazard ratio. 

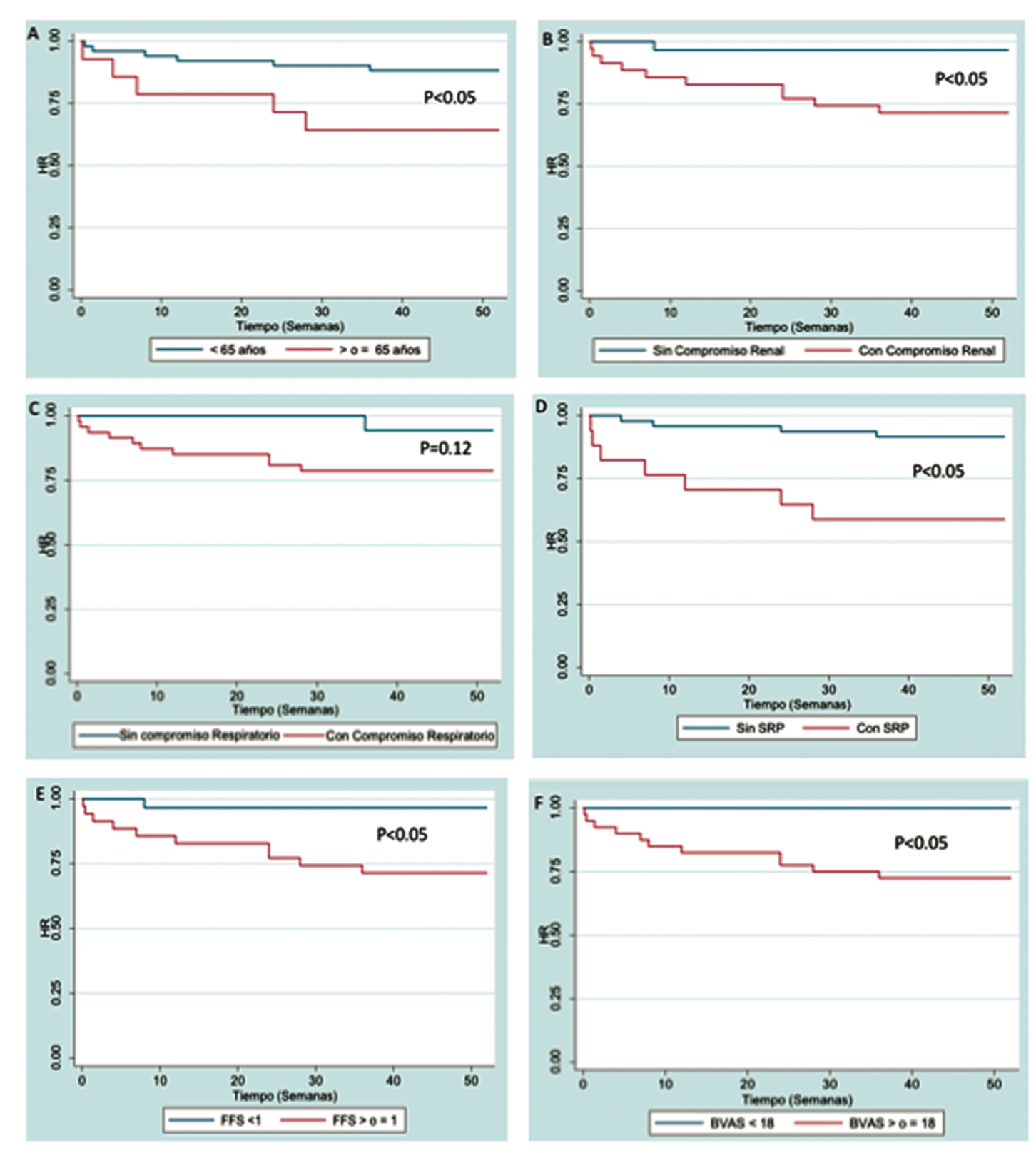

Figura 1. Curvas de sobrevida según: edad (A), compromiso renal (B), compromiso respiratorio (C), síndrome riñón pulmón (D), FFS (E) y BVAS (F). Curvas de sobrevida de Kaplan-Meier comparadas por log rank test, el p se muestra en cada cuadro.

\section{Discusión}

Existe poca información respecto al comportamiento de las VAA en pacientes latinoamericanos ya que la gran mayoría de los datos provienen de estudios europeos y en los últimos años asiáticos. Dado que la literatura respalda que existen diferencias étnicas en la presentación clínica de las $\mathrm{VAA}^{5,6}$ resulta interesante destacar algunos hallazgos de este trabajo. Respecto a las características demográficas, esta cohorte al igual que la anteriormente publicada en Chile ${ }^{8}$ fue más 
joven que lo descrito a nivel internacional ${ }^{1,10}$, con una mediana de edad de 53,5 años. Además hubo predominancia de mujeres, a diferencia de lo descrito en la literatura donde la proporción de ambos sexos es similar o con mayoría de hombres ${ }^{1,10-12}$.

En la presentación clínica de nuestra cohorte destaca la menor frecuencia de afección renal $(53,2 \%)$ respecto a lo descrito tanto nacional como internacionalmente. En cuanto al compromiso pulmonar, se observa un mayor porcentaje (72\%) que en las series europeas ${ }^{3,11-13}$, aunque similar a la cohorte china estudiada por Lai et al. ${ }^{10}$, con un número importante de pacientes con EPD y hemorragia alveolar. La afección ocular también fue frecuente, encontrando como manifestaciones principales epiescleritis y esclerouveitis.

Respecto del análisis de los auto-anticuerpos, lo más frecuente fue el patrón P ANCA y la presencia de MPO. Además, los pacientes mostraron valores más altos de reactantes de fase aguda que lo descrito, lo que traduciría un importante grado de inflamación sistémica. Estos hechos pueden explicar que el perfil clínico observado en esta cohorte tienda a ser más vasculítico que granulomatoso. En cuanto a las terapias utilizadas se evidencia un predominio de citotóxicos en relación a terapia de depleción de linfocitos B probablemente por ser, esta última, de uso más reciente y de alto costo. Hasta la fecha se sabe que para inducción de remisión a 6 meses, rituximab es no inferior a ciclofosfamida y es superior en caso de enfermedad recurrente. Los estudios no han demostrado diferencia en la mortalidad temprana entre una terapia y otra ${ }^{14,15}$.

La mortalidad al año fue de $16,9 \%$, cifra mayor que lo descrito en series europeas (entre 9\% y $11 \%)^{1,11}$, pero menor que en población asiática (entre un $21 \%$ y $23 \%)^{10,11}$. Los valores del FFS y del BVAS al diagnóstico también fueron similares a los datos publicados por Lai et al. ${ }^{10}$. Las principales causas de muerte fueron infecciones y vasculitis activa, y en la mayoría de los casos la muerte ocurrió antes de los tres meses del diagnóstico, semejante a lo informado por otros autores ${ }^{8,17}$.

Reportes de otros países han identificado como factores asociados a muerte al año la edad y el compromiso renal y pulmonar, este último en cohortes orientales ${ }^{10,17-19}$. En nuestra cohorte se encontró que el síndrome riñón pulmón, el compromiso oftalmológico, y un valor de FFS $>$ o igual a 1 fueron predictores independientes de muerte al año. La edad y el compromiso renal aparecieron como factores de riesgo en el análisis univariado, pero no se mantuvieron en el multivariado probablemente por el pequeño tamaño de la muestra. A pesar de que el síndrome riñón pulmón representa una falla orgánica grave $y$ muchas veces fulminante, no ha sido incluida en la descripción de las cohortes más numerosas. A diferencia de lo frecuentemente descrito, el compromiso oftalmológico fue de tipo no granulomatoso, lo que pudiese reflejar formas más severas de enfermedad en nuestra población. Aunque el FFS es una herramienta simple y útil para predecir mortalidad, no había sido evaluada previamente en pacientes chilenos.

Este es el primer estudio que evalúa factores asociados a mortalidad al año en pacientes con VAA en Chile y Latinoamérica. Antes de extrapolar las conclusiones al resto de la población local, se deben considerar entre las limitaciones de este análisis, que se trata de un estudio retrospectivo y con pacientes pertenecientes a un solo centro, que corresponde a un hospital universitario de alta complejidad.

En conclusión, en el perfil clínico de esta cohorte chilena tienden a predominar las manifestaciones secundarias a la capilaritis más que a la inflamación de tipo granulomatosa, con una alta frecuencia de compromiso pulmonar y predominio de MPO en la detección de ANCA por ELISA. Los factores asociados a mortalidad al año fueron el síndrome riñón pulmón, el compromiso oftalmológico y el valor de FFS $>$ o igual a 1 . Se requieren más estudios para establecer con mayor precisión el comportamiento clínico y los factores pronósticos de las VAA en nuestro país.

\section{Referencias}

1. Flossmann O, Berden A, De Groot K, Hagen C, Harper L, Heijl C, et al. Long-term patient survival in ANCA-associated vasculitis. Ann Rheum Dis 2011; 70 (3): 488-94.

2. Tan JA, Dehghan N, Chen W, Xie H, Esdaile JM, Avina-Zubieta JA, et al. Mortality in ANCA-associated vasculitis: a meta-analysis of observational studies. Ann Rheum Dis 2017; 76 (9): 1566-74.

3. Luqmani RA, Bacon PA, Moots RJ, Janssen B A, Pall A, Emery $\mathrm{P}$, et al. Birmingham Vasculitis Activity Score 
(BVAS) in systemic necrotizing vasculitis. QJM 1994; 87 (11): 671-8.

4. Guillevin L, Pagnoux C, Seror R, Mahr A, Mouthon L, Le Toumelin P. The Five-Factor Score revisited: assessment of prognoses of systemic necrotizing vasculitides based on the French Vasculitis Study Group (FVSG) cohort. Medicine (Baltimore) 2011; 90 (1): 19-27.

5. Watts RA, Mahr A, Mohammad AJ, Gatenby P, Basu N, Flores-Suárez LF. Classification, epidemiology and clinical subgrouping of antineutrophil cytoplasmic antibody (ANCA)-associated vasculitis. Nephrol Dial Transplant 2015; 30 (Suppl 1): i14-i22.

6. Pearce FA, Craven A, Merkel PA, Luqmani RA, Watts RA. Global ethnic and geographic differences in the clinical presentations of anti-neutrophil cytoplasm antibody-associated vasculitis. Rheumatology (Oxford) 2017; 56 (11): 1962-9.

7. Sreih AG, Mandhadi R, Aldaghlawi F, Khan A, Irshad V, Finn K, et al. ANCA-associated vasculitis in Hispanic Americans: an unrecognized severity. Clin Rheumatol 2015; 34 (5): 943-8.

8. Cisternas M, Soto L, Jacobelli S, Marinovic MA, Vargas A, Sobarzo E, et al. Manifestaciones clínicas de la granulomatosis de Wegener y la poliangeítis microscópica en Santiago-Chile, 1990-2001. Rev Med Chile 2005; 133 (3): 273-8.

9. Mukhtyar C, Lee R, Brown D, Carruthers D, Dasgupta B, Dubey S, et al. Modification and validation of the Birmingham Vasculitis Activity Score (version 3). Ann Rheum Dis 2009; 68 (12): 1827-32.

10. Lai QY, Ma TT, Li ZY, Chang DY, Zhao MH, Chen M. Predictors for mortality in patients with antineutrophil cytoplasmic autoantibody-associated vasculitis: a study of 398 Chinese patients. J Rheumatol 2014; 41 (9): 1849.

11. Eriksson P, Jacobsson L, Lindell A, Nilsson JA, Skogh $\mathrm{T}$. Improved outcome in Wegener's granulomatosis and microscopic polyangiitis? A retrospective analysis of 95 cases in two cohorts. J Intern Med 2009; 265 (4): 496-506.

12. Lane SE, Watts RA, Shepstone L, Scott DG. Primary systemic vasculitis: clinical features and mortality. QJM 2005; 98 (2): 97-111.

13. Solans-Laqué R, Fraile G, Rodríguez-Carballeira M, Caminal L, Castillo MJ, Martínez-Valle F, et al. Clinical characteristics and outcome of Spanish patients with ANCA-associated vasculitides: Impact of the vasculitis type, ANCA specificity, and treatment on mortality and morbidity. Medicine (Baltimore) 2017; 96 (8): e6083.

14. Specks U, Merkel PA, Seo P, et al. Efficacy of remission-induction regimens for ANCA-associated vasculitis. N Engl J Med 2013; 369 (5): 417-27.

15. Jones RB, Furuta S, Tearvaert JW, Hauser T, Luqmani RA, Morgan MD, et al. Rituximab Versus Cyclophosphamide in ANCA- associated renal Vascuilitis: 2 -year results of a randomised trial. Ann Rheum Dis 2015; 74 (6): 1178-82.

16. Mukhtyar C, Flossmann O, Hellmich B, Bacon P, Cid $\mathrm{M}$, Cohen-Tervaert JW, et al. Outcomes from studies of antineutrophil cytoplasm antibody associated vasculitis: a systematic review by the European League Against Rheumatism systemic vasculitis task force. Ann Rheum Dis 2008; 67: 1004-10.

17. Little MA, Nightingale P, Verburgh CA, Hauser T, De Groot K, Savage C, et al. Early mortality in systemic vasculitis: relative contribution of adverse events and active vasculitis. Ann Rheum Dis 2010; 69: 1036-43.

18. Yamagata K, Usui J, Saito C, Yamaguchi N, Hirayama $\mathrm{K}$, Mase $\mathrm{K}$, et al. ANCA-associated systemic vasculitis in Japan: clinical features and prognostic changes. Clin Exp Nephrol 2012; 16 (4): 580-8.

19. Murosaki T, Sato T, Akiyama Y, Nagatani K, Minota $\mathrm{S}$. Difference in relapse-rate and clinical phenotype by autoantibody-subtype in Japanese patients with anti-neutrophil cytoplasmic antibody-associated vasculitis. Mod Rheumatol 2017; 27 (1): 95-101. 